\title{
Surveying the Adaptations during the COVID-19 Outbreak in Turkish Radiotherapy Practice
}

\author{
Duygu SEZEN, ${ }^{1}$ (D) Uğur SELEK, ${ }^{1,2}$ (D) Eyüb Yaşar AKDEMiR, ${ }^{1}$ (I) Şükran ŞENYÜREK,' \\ (1) Nülifer KILIÇ DURANKUŞ, ${ }^{1}$ (D) Erkan TOPKAN, ${ }^{3}$ (1) Yasemin BÖLÜKBAŞI ${ }^{1,2}$
}

'Department of Radiation Oncology, Koc University Faculty of Medicine, İstanbul-Turkey

${ }^{2}$ Department of Radiation Oncology, University of Texas MD Anderson Cancer Center, Houston- $A B D$

${ }^{3}$ Department of Radiation Oncology, Baskent University Faculty of Medicine, İstanbul-Turkey

\begin{abstract}
OBJECTIVE
We aimed to assess how Turkish Radiation Oncology departments responded to the COVID-19 outbreak and to what extent adapted their routine practice per recent guidelines and recommendations.
\end{abstract}

\section{METHODS}

In late March 2020, a national survey was designed for Turkish radiation oncologists who were actively practicing and members of the Turkish Society for Radiation Oncology (TSRO). The survey created with an online survey program (www.SurveyMonkey.com), including 28 focused items. It was sent to all members of the TSRO on April $4^{\text {th }} 2020$.

\section{RESULTS}

Of responders, $17 \%$ noted to already complete these arrangements even before the first case announced. All of the responders declared that their team members, including the secretaries, nurses, and radiation therapists in the clinic, worked with appropriate protective equipment. While one-third of the centers (35\%) stated to implement a weekly shift, the other $1 / 3$ (29\%) chose to use daily shifts for social distancing and decreasing staff contact. Sixty-two percent of all responders indicated that multidisciplinary tumor boards discontinued, and the remaining $38 \%$ stated to continue with online video conferencing programs $(65 \%)$ or meeting in large rooms with a limited number of attendees social distancing (35\%).

\section{CONCLUSION}

Our survey revealed the importance of early precautions, communication by other Radiation Oncology Departments, following published guidelines during the COVID-19 pandemic, and the importance of using web-based applications for multidisciplinary tumor boards.

Keywords: COVID-19; radiation oncology; survey.

Copyright $\odot$ 2021, Turkish Society for Radiation Oncology

\section{Introduction}

Novel coronavirus disease 19 (COVID-19), characteristics of which were first described in Wuhan, China, rapidly led to a catastrophic event, and within 3 months, a pandemic was declared by the World Health Organization.[1] Unfortunately, as there has not yet been found any specific antiviral treatment or vaccination, prevention seems to be the best strategy to minimize the impact of outbreaks. Almost $20 \%$ of the infected people present with severe or critical clinical manifestation(s) and might need intensive care. [2] In oncological basis, this clinical scenario could be more complicated for cancer patients, who could 
have several risk factors such as multiple comorbidities, compromised immune system, and being elderly. COVID-19 incidence has turned out to be augmented and devastating in cancer patients and survivors. $[3,4]$ Therefore, particular attention has been recommended to these frail patients in terms of isolation in the social areas and hospitals while consciously continuing their necessary oncological care due to the natural history of cancer.

During the outbreak, almost all radiation oncology departments have become inherently an unsafe environment of viral transmission for both patients and staff, due to crowded waiting rooms, daily treatments over weeks, shared liner accelerators, and relatively frequent contacts of patients with radiotherapists, nurses or radiation oncologists. Worldwide radiotherapy centers have struggled with strict measures to stay operative, while early practical recommendations based on initial experiences with the COVID-19 outbreak were published.[5-8] Recently moreover, ASTRO, ESTRO, and several other groups have suggested disease sitespecific guidelines for radiotherapy delivery, and the Turkish Society for Radiation Oncology (TSRO) actively communicated with its members through emails and web-conferences.[9-15]

Just after the first case reported in Turkey on March 11,2020, The Turkish Ministry of Health reacted quickly and subsequently took some series of measures, while the burden of COVID-19 outbreak on the health-care system became more visible in a relatively short period. When general recommendations and guidelines were emerging, most Turkish radiotherapy centers adapted with adjustments in line with announced precautions. We aimed to assess how Turkish Radiation Oncology departments responded to the COVID-19 outbreak and to what extent adapted their routine practice per recent guidelines and recommendations.

\section{Materials and Methods}

In late March 2020, we designed a national survey aiming to capture the effects of COVID-19 outbreak on the clinical practice of radiation oncology departments in Turkey for Turkish radiation oncologists who were actively practicing and members of the TSRO. The survey was created via an online survey program (www. SurveyMonkey.com), including 28 focused items. It was distributed amongst the members of the TSRO on April 4, 2020, through email notification and a link posted on the TSRO website and asked to be completed in the next 3 weeks. The time required to complete the survey was approximately $10 \mathrm{~min}$. Koc University Scientific Research Ethics Board approved the study before any data collected.

In the first section of the survey, demographics, and line of work data (i.e., age, gender, type of hospital, and professional title of the physicians) were questioned to identify factors that could influence responses. Hospital types were divided into four groups; university hospitals, state training, and research hospitals, public/city hospitals, and private hospitals, and the academic titles were within three groups professor/associate professor, radiation oncologist/assistant professor, and radiation oncologist resident.

In the second section of the survey, incorporation of the previous clinical routine process into new pandemic burden was evaluated along with the timing and extent; the need to acquire input from other radiation oncology clinics related with arrangements, the start date of precautions, the protective equipment usage, any changes in schedules or shifts, the social distance in waiting room and treatment device, and the continuity of tumor boards.

In the third section of the survey, re-arrangements performed for the treatment and follow-up in the pandemic were evaluated, such as postponing of treatment/follow-up, modifications in prescriptions/ fractionation, the recommendation of prophylaxis for COVID, approach to patients suspected/diagnosed with COVID.

\section{Results}

Our survey was responded by 83 radiation oncologists (15\% of all active Turkish Radiation Oncologists). The median age was 45 years (25-70), and the male/female ratio was 1:2. The respondents' distribution was as follows: $41 \%$ university hospitals, $36 \%$ Ministry of Health hospitals, and $23 \%$ private hospitals. Of the responders, $32 \%$ had academic affiliations (professors or associate professors), while $12 \%$ were residents. Table 1 demonstrates the questions and response rates.

\section{Department Re-arrangements}

The response has demonstrated that the communication rate with other departments for exchanging considerations related with COVID precautions was $71 \%$. Almost 2/3 of the responders (59\%) stated that all re-arrangements of the clinical process were performed in the same week of the first case in Turkey; while $17 \%$ of re- 
Table 1 A national survey was design to capture the effects of COVID-19 outbreak on the clinical practice of radiation oncology departments

\section{First part}

1. In which hospital is your primary practice/work?
a) University Hospital
b) Ministry of Health Training and Research Hospital
c) Ministry of Health Public/City Hospital
d) Private Hospital

2. What is your current profession or position?

$\begin{array}{ll}\text { a) Professor/Associate Professor } & 32.1 \\ \text { b) Radiation Oncologist/Assistant Professor } & 55.6 \\ \text { a) Radiation Oncology Resident } & 12.3\end{array}$

3. What is your gender?
a) Female
b) Male
4. What is your age?

Second part

5. Do you need to acquire information from other radiation oncology clinics related with arrangements for COVID?

a) Yes

b) No

6. When did you perform the first arrangements for pandemic in your clinic?

a) Before the first case reported in Turkey ( $12^{\text {th }}$ March 2020)

b) In the same week the first case reported in Turkey (12 $2^{\text {th }}$ March 2020$)$

c) After the first case seen in Turkey (March 12, 2020)

d) Two weeks after the first case seen in Turkey (March 12, 2020)

7. Do you use any the protective equipment in your clinic for prevention?
a) Yes
b) No

8. Do secretaries use the protective equipment?
a) Yes
b) No

9. Do radiation therapists use protective equipment?

a) Yes

b) No

10. Do nurses use protective equipment?
a) Yes
b) No

11. How do you coordinate work hours and staff schedules in your department?
a) Weekly shift
b) Daily shift
c) Shift every 3-days
d) Other

12. Do tumor boards (multidisciplinary meetings) continue in your hospital?
a) Yes
b) No

13. If yes, how do tumor boards (multidisciplinary meetings) perform?
a) Video conference
b) In the hall by social distancing

14. Do you take any precautions in the waiting rooms to reduce the access of people?
a) Yes
b) No

15. Do you you extend the time interval between patients' appointment?
a) Yes
61.4
b) No 
Table 1 Cont.

Third part

Response

rate (\%)

16. Do you postpone the appointments of follow-up patients?
a) Yes
97.6
b) No

17. Please select the most important criteria that you consider to postpone the appointments of follow-up patients?
a) Initial stage
10.4
b) Primary of disease
41.6
c) Disease-free survival
40.3
d) Patient age
e) Patient's anxiety
18. Do you continue to weekly-see visits based on the new complaint or symptoms?
a) Yes
91.6
b) No

19. Do you postpone the treatment start of any simulated patients when the pandemic declared?
a) Yes
41.5
b) No

20. Do you postpone the treatment of new consultations after the pandemic declared?
a) Yes
b) No

21. Please select the patient group or diagnose that you will postpone the treatment?
a) Adjuvant treatment (up to the maximum time recommended by guidelines)
b) Prostate treatment (adjuvant/salvage/definitive)
c) 65-years-old and older patients
d) Multiple comorbidities
e) COVID suspected symptoms or radiographic sign
f) Palliative treatment (excluding spinal cord compression, vena cava superior syndrome, bleeding)
g) Inadequate hospital conditions
h) Others

22. Do you call your patients who has finished their treatment at the pandemic period for early follow-up at 6th week?
a) Yes
b) No

23. Do you consider modifying the fraction schemes of the patients under treatment according to ASTRO, ESTRO and TSRO recommendations after the declaration of pandemic?
a) Yes
b) No

24. If you modify; which patient group do you adjust fraction dose and schemas?
a) Prostate cancer
b) Palliative treatment
c) Breast cancer
d) Head and neck cancer
e) Central nervous system cancer
f) Pediatric cancer
g) Sarcoma
h) Skin cancer
i) Rectal cancer
j) Lung cancer
k) Bladder cancer
l) Stomach cancer

25. Do you prefer hypofraction schemes for new patient planning?
a) Yes
b) No 
Table 1 Cont.

\section{Third part}

Response

rate (\%)

26. Do you recommend any drugs or supplements for prophylaxis of COVID-19?
a) Hydroxiclorochine
b) Azithromycin
c) Vitamin supplement
32.5
d) None
55.4
e) Others

27. Do you have any COVID-19 suspected patients during radiation therapy?
a) Yes
19.3
b) No
80.7

28. How do you act when your patients has COVID-19 suspicion during radiation therapy?
a) Give a break until getting a clarification of the status
b) Continue to treatment with taking precautions
3.6
c) No patient
78.3

TSRO: Turkish society for radiation oncology

sponders noted to already complete these arrangements even before the first case announced. All of the responders declared that their team members, including the secretaries, nurses, and radiation therapists in the clinic, worked with appropriate protective equipment. While one-third of the centers (35\%) stated to implement a weekly shift, the other $1 / 3$ (29\%) chose to use daily shifts for social distancing and decreasing staff contact.

Sixty-two percent of all responders indicated that multidisciplinary tumor boards discontinued, and the remaining $38 \%$ stated to continue with online video conferencing programs $(65 \%)$ or meeting in large rooms with a limited number of attendees social distancing (35\%). Almost all participants declared to adopt regulations to maintain social distancing in the waiting rooms.

\section{Patients Treatment and Follow-up Data}

At the beginning of pandemic, $41 \%$ of the responders decided to defer the start of the treatment of already simulated patients, and $62 \%$ rearranged the sequencing of radiotherapy in patients with indications at the consultation to postpone the start of radiotherapy (Fig. 1).

Eighty-one percent of the responders stated to revise fractionation schemas according to published ESTRO, ASTRO, and TSRO recommendations, and most common sites found reasonable to be modified prostate cancer (34\%), palliative cases (31\%), and breast cancer (23\%). The majority (89\%) noted to prefer prescribing hypofractionated regimens for new patients.

An extended gap between treatment time slots in linear accelerators for patients under treatment was

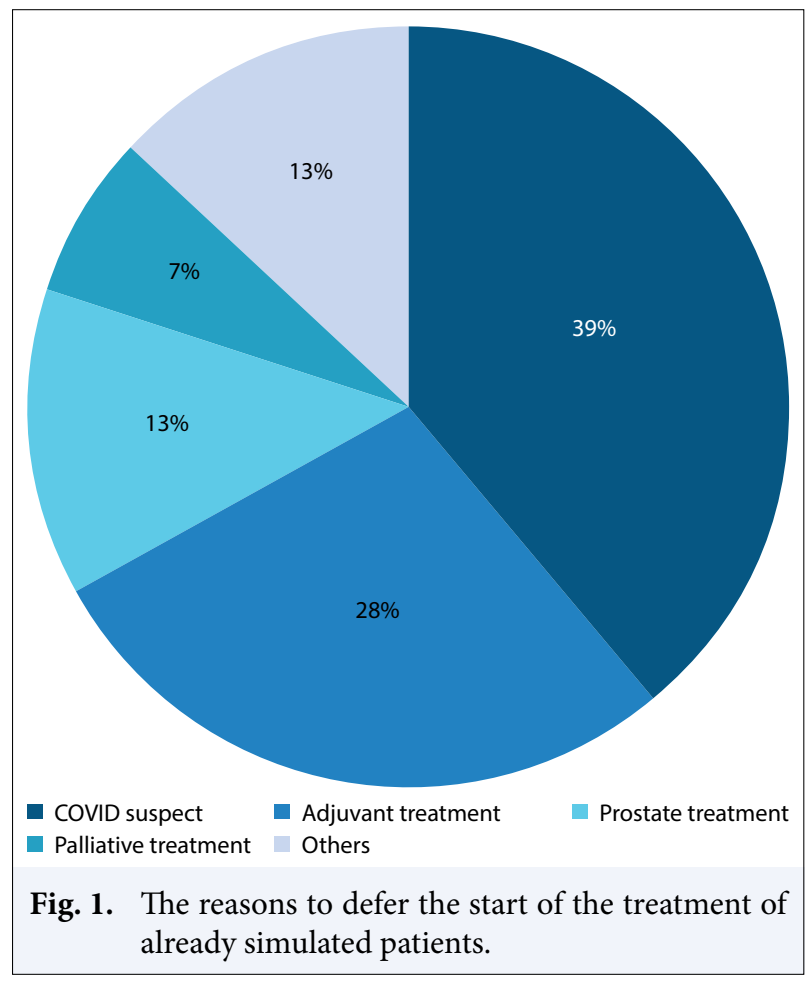

noted to be implemented by $61 \%$ of the responders. Ninety-two percent of the responders affirmed to carry out weekly see visits of their patients under treatment if presented with a new symptom or complaint. Almost all the responders (98\%) postponed routine follow-up appointments of previously treated patients if there is no complaints, while $88 \%$ of the responders noted to cancel the first routine follow-up after the recent completion of radiotherapy (Fig. 2). 


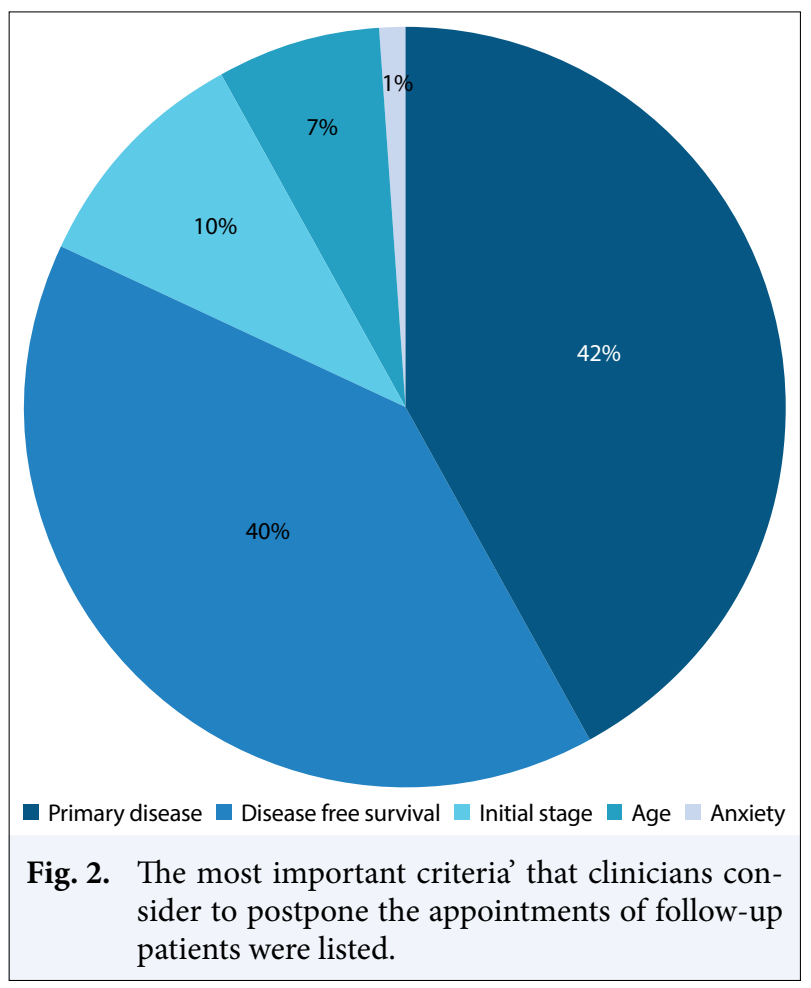

The responders were asked whether to recommend any medications for COVID-19 prophylaxis, and 55\% declared that they did not, while 32\% recommended empiric vitamin supplements.

$1 / 5(19 \%)$ of the responders declared that they had patients under treatment carrying suspicion for COVID-19 infection; of these responders, $80 \%$ declared to give treatment break during radiotherapy, and $20 \%$ continued treatment with additional precautions of extra clothing, gloves, goggles, and masks.

\section{Discussion}

COVID-19 has been dramatically changing our world as well as radiation oncology's practice. Our survey is to observe the necessary adaptations performed in this term related with this outbreak in Turkish radiation oncology practice following prudent country-based surveys or evaluations.[16-18] In our study, Turkish radiation oncologists sound to be in a consensus on many of the significant issues such as postponing new treatment starts and follow-up appointments, prescription of hypofractionated schemes, using protective equipment, and managing COVID-19 positive or suspected patients.

There are several reasons for the emergence of this consensus; first of all, before the COVID-19 outbreak in our country, we had the chance to take crucial lessons from other countries, especially Italy's experience. [5] Second, TRSO proactively communicated with its members through frequent emails informing about the first published reports in the literature and organizing dedicated interactive webinars comprising basic social distancing, protective measures, and options for hypofractionation before and during the time of the survey. Third, radiation oncologists also networked and discussed the management of this course on social media (WhatsApp, and telegram groups across the country), which also essentially has been implementing the significance of virtual communication. Those, as mentioned above, highly active communication network could be underlined by the response of our responders who are willing to gather information from other clinics $(71 \%)$ in terms of the process.

At the beginning of the outbreak, all major institutions were announced as pandemics hospitals by the Ministry of Health, and hospitals judiciously tried to find ways to reduce their routine patient volumes in order to avoid possible exaggerated burden with COVID. This intent was pronounced by almost $3 / 4$ of our responders $(71 \%)$ preferring hypofractionated schemes over conventional ones according to encouraging diseasesite specific guidelines or recommendations initially by TRSO, ASTRO, ESTRO, and other groups. $[9-15,19]$ Most part (88\%) of the preference for hypofractionation was consisted of prostate and breast cancer, besides palliative treatments, which could be rationalized based on the prevailing trend in recent years of many Turkish radiation oncologists already employing shortened schemes in these sites based on a large body of the evidence. In Italy, $73.6 \%$ of the responders implemented the extensive use of hypofractionated regimens, similar to our practical attitude. Nevertheless, we could not interpret the rest of the countries about how they have adapted hypofractionation from their surveys. $[17,18,20]$

In the lack of evidence-based prophylaxis with medication and in the presence of excessive fear to get sick with COVID-19, we asked our colleagues whether they are recommending any prophylaxis with any drugs or supplements and interestingly revealed that $32 \%$ of them recommended vitamin supplements while over the half of the responders, did not recommend any medication (e.g., hydroxychloroquine). We believe our responders could fight with the social distress of requesting prophylaxis, especially in the absence of evidence that was pointed out lately.[21,22]

The patient, with confirmed-COVID-19 infection or with just symptoms lacking a positive test, needs to 
be considered a possible health threat for the safety of radiation oncology staff, as well as for the other patients and families, in RO departments; therefore, precautions are necessary to decrease the transmission risk. In Spain, $78 \%$ of the COVID positive patients were reported to complete their radiotherapy courses without any major events,[20] while in Italy, the clinical routine was mainly encouraging to interrupt the treatment. [16] Among our cohort of Turkish radiation oncologists, the tendency was to allow a break during treatment and only $20 \%$ of patients with COVID- 19 were treated without any break with extra caution of clothing, gloves, Goggles, and masks.

We can tell from our colleague's responses that almost all of the radiotherapy departments performed rotations in personnel workflow, mostly using weekly or daily shifts, followed by 2-weekly or three-daily shifts depending on the eligibility of the staff and the workload as emphasized by many recent reports.[18,20,2325] Concerning using of personnel protective equipment (PPE) by staff, our survey showed unity in favor of the adoption of necessary PPE. Other recent surveys were consistent with ours, except a shortage of some of these items in the previous report.[16-18]

Unfortunate was the documentation of discontinuation of multidisciplinary tumor boards by $62 \%$ of our colleagues in their institutions in this survey, while the tumor boards were reported to continue by $38 \%$ of participants without any break with online video conferencing programs $(65 \%)$ or meeting in large rooms with a limited number of attendees with social distance distancing (35\%). As this pandemics was unexpected and sudden, many institutions could not adapt secure multidisciplinary online connection options as necessarily fast as possible at the time of this survey. However, most were evaluating affordable and secure options to defeat the infection risk in multiple meetings per week to continue necessary interdisciplinary interaction, which would probably be a part of long term telemedicine efforts among physicians and between physicians and patients.

\section{Conclusion}

Our survey revealed the importance of early precautions and communication by the Radiation Oncology Departments during the COVID-19 pandemic. Mainly adapting hypofractionation schemas and personal protection procedures provide the continuation of radiotherapy service for cancer patients while keeping the healthcare staff safe from possible COVID-19 dissemi- nation. This experience gained from the unexpected rush of pandemics will hopefully help to build a more prepared radiation oncology system for any disasters ahead, along with the effort to continue interaction via pandemics taught measures including telemedicine.

Peer-review: Externally peer-reviewed.

Conflict of Interest: All authors declared no conflict of interest.

Ethics Committee Approval: The study was approved by the Koc University Scientific Research Ethics Committee (No: 2020.110.IRB1.021, Date: 25/03/2020).

Financial Support: None declared.

Authorship contributions: Concept - D.S., U.S., E.Y.A., S..S., N.K.D., E.T., Y.B.; Design - D.S., N.K.D., Ş.Ş., E.Y.A.; Supervision - U.S., Y.B., E.T.; Funding - None; Materials - N.K.D.; Data collection and/or processing - E.Y.A., Ş.Ş., D.S.; Data analysis and/or interpretation - Y.B., S..Ş.; Literature search - Ş.Ş., E.Y.A., D.S.; Writing - D.S., Y.B.; Critical review - E.T., U.S.

\section{References}

1. Chen N, Zhou M, Dong X, Qu J, Gong F, Han Y, et al. Epidemiological and clinical characteristics of 99 cases of 2019 novel coronavirus pneumonia in Wuhan, China: A descriptive study. Lancet 2020;395(10223):507-13.

2. Wu Z, McGoogan JM. Characteristics of and important lessons from the coronavirus disease 2019 (COVID-19) outbreak in China: Summary of a report of 72314 cases from the Chinese center for disease control and prevention. JAMA 2020;323(13):1239-42.

3. Liang W, Guan W, Chen R, Wang W, Li J, Xu K, et al. Cancer patients in SARS-CoV-2 infection: A nationwide analysis in China. Lancet Oncol 2020;21(3):3357.

4. Zhang L, Zhu F, Xie L, Wang C, Wang J, Chen R, et al. Clinical characteristics of COVID-19-infected cancer patients: A retrospective case study in three hospitals within Wuhan, China. Ann Oncol 2020;31(7):894-90.

5. Filippi AR, Russi E, Magrini SM, Corvo R. Letter from Italy: First practical indications for radiation therapy departments during COVID-19 outbreak. Int J Radiat Oncol Biol Phys 2020;107(3):597-9.

6. Krengli M, Ferrara E, Mastroleo F, Brambilla M, Ricardi $U$. Running a radiation oncology department at the time of coronavirus: An Italian experience. Adv Radiat Oncol 2020;5(4):527-30.

7. Portaluri M, Tramacere F, Portaluri T, Gianicolo EA. Southern Italy: How the supply of radiation therapy, 
patient outcomes, and risk to health care providers have changed during the COVID-19 pandemic. Adv Radiat Oncol 2020;5(4):597-600.

8. Rivera A, Ohri N, Thomas E, Miller R, Knoll MA. The impact of COVID-19 on radiation oncology clinics and cancer patients in the United States. Adv Radiat Oncol 2020;5(4):538-43.

9. Coles CE, Aristei C, Bliss J, Boersma L, Brunt AM, Chatterjee $S$, et al. International guidelines on radiation therapy for breast cancer during the COVID-19 pandemic. Clin Oncol 2020;32(5):279-81.

10. Guckenberger M, Belka C, Bezjak A, Bradley J, Daly ME, DeRuysscher D, et al. Practice recommendations for lung cancer radiotherapy during the COVID-19 pandemic: An ESTRO-ASTRO consensus statement. Radiother Oncol 2020;146:223-9.

11. Romesser PB, Wu AJ, Cercek A, Smith JJ, Weiser M, Saltz L, et al. Management of locally advanced rectal cancer during the COVID-19 pandemic: A necessary paradigm change at memorial sloan kettering cancer center. Adv Radiat Oncol 2020;5(4):687-9.

12. Thomson DJ, Palma D, Guckenberger M, Balermpas P, Beitler JJ, Blanchard P, et al. Practice recommendations for risk-adapted head and neck cancer radiotherapy during the COVID-19 pandemic: An ASTROESTRO consensus statement. Int J Radiat Oncol Biol Phys 2020;107(4):618-27.

13. Wu AJ, Rimner A, Shepherd AF, Gelblum DY, Shaverdian N, Yorke E, et al. Thoracic radiation therapy during COVID-19: Provisional guidelines from a comprehensive cancer center within a pandemic epicenter. Adv Radiat Oncol 2020;107(4):618-27.

14. Yahalom J, Dabaja BS, Ricardi U, Ng A, Mikhaeel NG, Vogelius IR, et al. ILROG emergency guidelines for radiation therapy of hematological malignancies during the COVID-19 pandemic. Blood 2020;135(21):182932.

15. Yerramilli D, Xu AJ, Gillespie EF, Shepherd AF, Beal K, Gomez D, et al. Palliative radiotherapy for oncologic emergencies in the setting of COVID-19: Approaches to balancing risks and benefits. Adv Radiat Oncol 2020;5(4):589-94.

16. Jereczek-Fossa BA, Pepa M, Marvaso G, Bruni A, di
Monale e Bastia MB, Catalano G, et al. COVID-19 outbreak and cancer radiotherapy disruption in italy: Survey endorsed by the italian association of radiotherapy and clinical oncology (AIRO). Radiother Oncol 2020;149:89-93.

17. ASTRO. COVID-19's Impact on Radiation Oncology Initial Results of a Nationwide Physician Survey. United States: ASTRO; 2020.

18. Tamari K, Nagata Y, Nishiki S, Nakamura S, Ogawa K, Uno T. Nationwide survey of COVID-19 prevention measures in Japanese radiotherapy departments via online questionnaire for radiation oncologists. Radiother Oncol 2020;149:219-21.

19.Zaorsky NG, Yu JB, McBride SM, Dess RT, Jackson WC, Mahal BA, et al. Prostate cancer radiation therapy recommendations in response to COVID-19. Adv Radiat Oncol 2020;5(4):659-65.

20.Suárez V, Moreno-Olmedo E, Pérez M, González EJ, Rivas D, Fusco J, et al. Spanish risk management framework across 17 radiation oncology centers during COVID-19 pandemic. Radiother Oncol 2020;148:267-9.

21. Boulware DR, Pullen MF, Bangdiwala AS, Pastick KA, Lofgren SM, Okafor EC, et al. A randomized trial of hydroxychloroquine as postexposure prophylaxis for covid-19. N Engl J Med 2020;383:517-25.

22. Cohen MS. Hydroxychloroquine for the prevention of Covid-19-searching for evidence. N Engl J Med 2020;383(6):585-6.

23. Wei W, Zheng D, Lei Y, Wu S, Verma V, Liu Y, et al. Radiotherapy workflow and protection procedures during the Coronavirus Disease 2019 (COVID-19) outbreak: Experience of the Hubei Cancer Hospital in Wuhan, China. Radiother Oncol 2020;148:203-10.

24. Simcock R, Thomas TV, Estes C, Filippi AR, Katz MS, Pereira IJ, et al. COVID-19: Global radiation oncology's targeted response for pandemic preparedness. Clin Transl Radiat Oncol 2020;22:55-68.

25. Combs SE, Belka C, Niyazi M, Corradini S, Pigorsch S, Wilkens J, et al. First statement on preparation for the COVID-19 pandemic in large German Speaking University-based radiation oncology departments. Radiat Oncol 2020;15(1):74. 